\title{
Systematic and Steered Evaluation to Detect Early-onset Musculoskeletal Involvement in Psoriasis Patients
}

\author{
Minerva Gómez-Flores ${ }^{1}$, Jorge Ocampo-Candiani ${ }^{1}$, Rubicela Garza-Garza ${ }^{1}$, Diana E. Flores-Alvarado², \\ David Vega-Morales², Ana C. Arana-Guajardo², Jorge A. Esquivel-Valerio², Lorenia B. de la Cruz-Becerra², \\ Karina Silva-Luna², Mario R. García-Pompermayer ${ }^{2}$ and Mario A. Garza-Elizondo² \\ ${ }^{1}$ Servicio de Dermatología, ${ }^{2}$ Servicio de Reumatología, Hospital Universitario "Dr. José Eleuterio González," Universidad Autónoma de Nuevo \\ León, Mexico
}

\begin{abstract}
Introduction: The aim of this study is to evaluate a systematic and steered protocol to detect musculoskeletal involvement in psoriasis patients. Materials and Methods: We made a comparative, cross-sectional, observational, descriptive, and analytical study. We included Mexican mestizo patients over 18 years old with psoriasis. The evaluation protocol included three steps: first, a clinical evaluation (Toronto psoriatic arthritis Screening [ToPAS] questionnaire and inflammatory low back pain [ILB] searching by Assessment of SpondyloArthritis Society criteria); second, patients were categorized according to their symptoms, and third, an image evaluation (OMERACT and Madrid Sonographic Enthesitis Index [MASEI] musculoskeletal ultrasound evaluation and sacroiliac and lumbar magnetic resonance imaging) was performed. Results: We included 75 patients, 33 (44\%) of whom were women. Mean age was 44.6 (standard deviation 14.5) years. We found 16 (21.3\%) symptomatic patients. Group 1: nine of 11 patients had findings suggestive of inflammatory joint disease. Group 2: only one of seven patients with ILB had inflammatory changes in the sacroiliac joints. Group 3: from the remaining ToPAS and ILB-negative patients, 15 of 27 were detected to have enthesopathy, with a score of MASEI $\geq 20$ by ultrasound. The interobserver kappa index for detecting enthesopathy by MASEl index was 0.37. Despite the loss of 33 patients, the systematic protocol detected 26 of 75 patients with musculoskeletal involvement. Conclusion: This systematic and steered approach is useful for hidden inflammatory musculoskeletal involvement in psoriasis patients.
\end{abstract}

Key words: Psoriasis. Psoriatic arthirits. Diagnosis.

\section{Introduction}

Psoriasis is a multisystem polygenic disease, inflammatory, chronic, and mediated by activated T-lymphocytes, predominantly involving the skin, joints, and enthesis.

The prevalence of the joint involvement in psoriasis is not easy to determine due to the absence of visible inflammatory signs and subclinical involvement. Finally, it can cause permanent damage and affects from $7 \%$ to $40 \%$ of patients with psoriasis. It is estimated that $25 \%$ of patients who suffer from articular involvement are underdiagnosed, although a systematic review estimated $15 \%{ }^{1}$. Early detection of joint conditions and treatment has been shown to improve the prognosis of patients with this inflammatory arthropathy ${ }^{1,2}$.

Screening questionnaires have been used to identify psoriatic arthritis (PSA) in patients with psoriasis ${ }^{3}$. The use of clinical questionnaires and imaging methods as

\section{Correspondence:}

David Vega-Morales

E-mail: drdavidvega @yahoo.com.mx
Available online: $30-05-2018$

Date of reception: 08-08-2017

Date of acceptance: 05-12-2017

DOI: 10.24875/RMU.M18000006
Medicina Universitaria. 2018;20(1):13-21 www.medicinauniversitaria.org

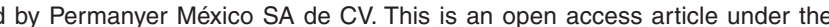
CC BY-NC-ND license (http://creativecommons.org/licenses/by-nc-nd/4.0/). 
a systematic search of joint disease in patients with psoriasis with or without musculoskeletal symptoms has not been evaluated ${ }^{1}$.

Our question is if the implementation of a systematic and steered evaluation protocol will be able to detect musculoskeletal inflammatory involvement in patients with psoriasis.

\section{Materials and Methods}

This project was approved by our IRB with the registry number DE12-005. It was a comparative cross-sectional, observational, descriptive, and analytical study. The study took place from July 2012 to October 2014. We included all Mexican mestizo patients over 18 years old with a clinical and/or histological diagnosis of psoriasis in any of its clinical varieties and at all the degrees of severity from the Dermatology Clinic at the University Hospital who agreed to participate in the study and sign the informed consent form. We excluded patients with a previous diagnosis of PsA and those who met criteria for some other autoimmune rheumatologic entity with musculoskeletal involvement. The patients who were unable to read or write were also excluded from this study.

The systematic search protocol is described below and diagrammed in figure 1. The evaluation protocol included three steps: first, a clinical evaluation; second, the patients were categorized according to their symptoms, and third, an image evaluation.

\section{History and physical examination}

All patients underwent a complete medical history and physical examination with a dermatological and rheumatological review of systems.

The severity of psoriasis was recorded by the Psoriasis Area and Severity Index ${ }^{4}$. Furthermore, its impact on quality of life was recorded by the Psoriasis Disability Index ${ }^{5}$ and the Dermatology Life Quality Index ${ }^{6}$. Nail involvement was recorded by the Nail Psoriasis Severity Index?

\section{Clinical evaluation for joint and entheseal involvement}

To determine the clinical suspicion of arthritis, the Toronto PsA Screening Questionnaire (ToPAS) was applied $^{8}$. Positive ToPAS was defined as $\geq 8$ score. To determine the presence of inflammatory low back pain (ILB), we used the international Assessment of SpondyloArthritis Society (ASAS) criteria $^{9}$. Physical examination was performed by a certificated rheumatologist (DEFA). The presence of probable enthesopathy was registered with the Maastricht Ankylosing Spondylitis Enthesitis Score ${ }^{10}$.

\section{Image assessment}

Blinded to clinical evaluation, certified ultrasonographers performed an articular and entheseal ultrasound with an Esaote ${ }^{\circledR}$ My Lab 70 XVG with a linear transducer at $6-18 \mathrm{MHz}$. Power Doppler adjustments were standardized with a pulse repetition frequency of $700 \mathrm{MHz}$, a gain of $50 \mathrm{~dB}$, and a low filter wall.

The ultrasound findings of joint involvement were described in the terminology proposed by the Outcome Measures in Rheumatology (OMERACT) group ${ }^{11}$. The ultrasound variables included: bone erosion, synovial fluid, synovial hypertrophy, tenosynovitis, and enthesopathy.

The Madrid Sonographic Enthesitis Index (MASEI) was used to generate a global scale to reflect the severity and extension of entheseal abnormalities in all sites scanned on both sides in each subject. Total MASEI score was categorized as follows: inflammatory MASEl: changes including entheseal thickening, structural changes, bursitis, and vascularization and damaged MASEl: chronic damage including calcifications, enthesophytes, and erosions ${ }^{12}$. A cutoff of $\geq 20$ MASEI was used to determine the significant presence of enthesopathy. Furthermore, the presence of power Doppler signals (PDUSs) in any enthesis was evaluated.

The articular ultrasound was made by one ultrasound certified rheumatologist, and the entheseal ultrasound was evaluated by two ultrasound certified rheumatologists.

The sacroiliac and lumbar spine magnetic resonance imaging (MRI) was performed on a 1.5T MR imaging system (GE, Wisconsin, USA). The basic protocol consisted of coronal and axial oblique fast spin-echo T1-weighted sequences to detect structural changes, and coronal and axial oblique short inversion time inversion recovery or fat-saturated fast spin-echo T2-weighted sequences to detect acute inflammatory changes.

\section{Evaluation protocol}

After the history and physical examination, the patients were divided into three groups: Group 1 , the patients with a positive ToPAS questionnaire; Group 2, the patients with ILB; and Group 3, the patients with a negative ToPAS and an absence of ILB. The patients could belong to Groups 1 and 2 synchronously. 


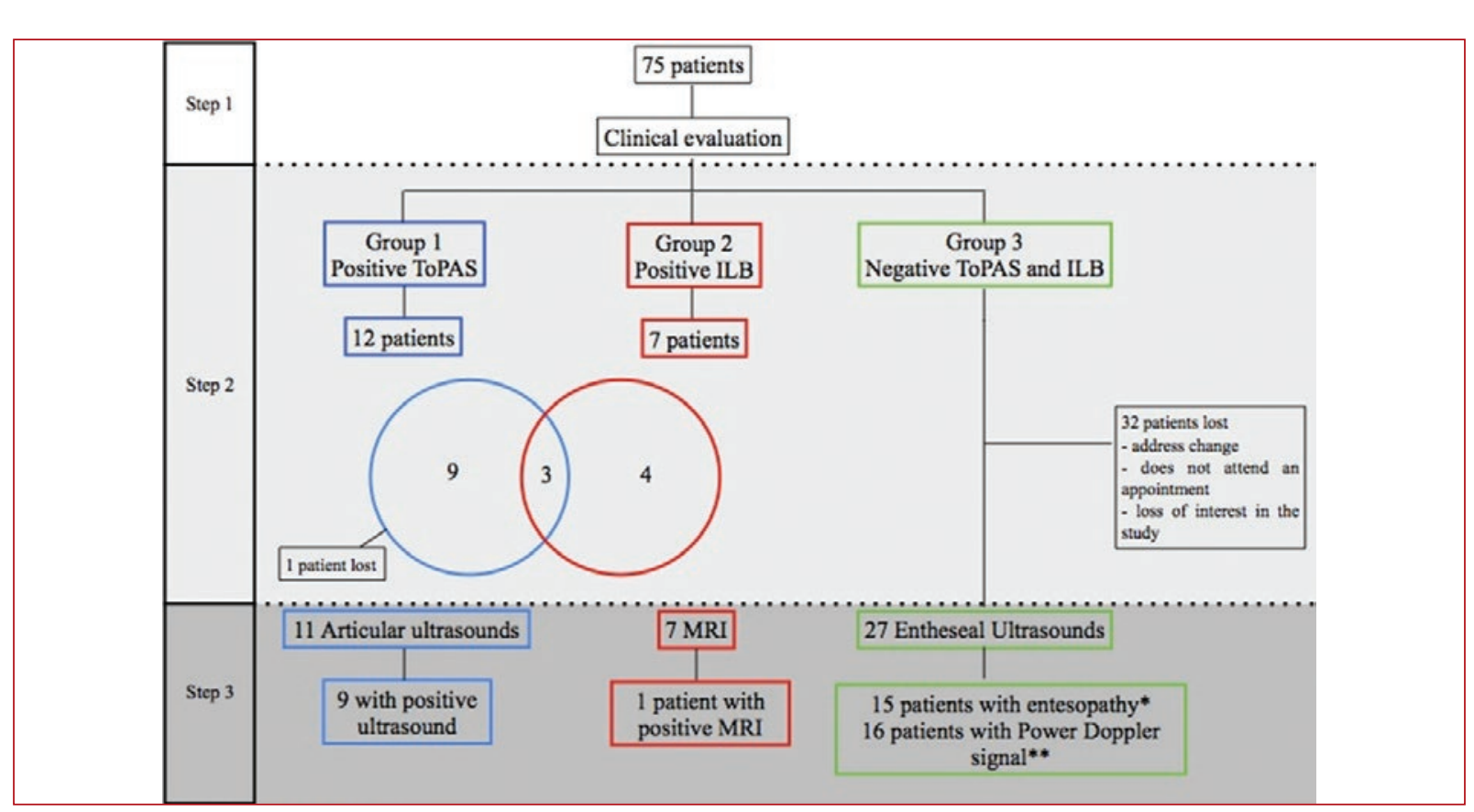

Figure 1. Evaluation protocol

*Enthesopathy MASEI > 20, **in any enthesis

ToPAS: Toronto Psoriatic Arthritis Screening Questionnaire, ILB: inflammatory low back pain, MASEI: Madrid Sonographic Enthesitis Index

Finally, the patients in Group 1 with positive ToPAS underwent a musculoskeletal ultrasound. The patients in Group 2 with ILB underwent both sacroiliac and lumbar spine MRI. Moreover, the patients in Group 3 with negative ToPAS questionnaire and negative ILB underwent an entheseal ultrasound.

\section{Statistical analysis}

Statistical analysis was performed using SPSS version 20.0 (SPSS, Chicago, IL, USA). Quantitative variables were presented as the mean (standard deviation [SD]) and median (interquartile range), depending on their Gaussian distribution. Categorical variables were presented as absolute frequencies and percentages. Kappa statistic and correlation coefficients (Spearman's rho) for analysis between two entheseal ultrasound evaluators were used. A value of $p<0.05$ was considered statistically significant.

\section{Results}

We included 75 patients, 33 (44\%) of whom were women. Mean age was 44.6 (SD 14.5) years. All 75 patients underwent a complete medical history and physical examination with a dermatological and rheumatological review of systems.

Table 1 summarizes the demographic characteristics of the entire cohort. In figure 1, we describe the groups according to the presence (or absence) of symptoms. We found $16(21.3 \%)$ symptomatic patients.

\section{Evaluation protocol}

Group 1: from this group, only 11/12 patients were evaluated. One patient did not attend the appointment. Nine patients had findings suggestive of inflammatory joint disease (Table 3).

Group 2: seven patients with ILB were detected. An $\mathrm{MRI}$ was conducted on all seven patients. Only one patient had inflammatory changes in the sacroiliac joints (Table 4).

Group 3: of the remaining patients, there were 59 negative to ToPAS and ILB. Of these, only 27 of 59 patients were available to perform an entheseal ultrasound (Fig. 1). We detected enthesopathy in 15 patients, with a MASEl score of $\geq 20$. The interobserver kappa index for detecting enthesopathy by MASEl index was 0.37 . Comparison of the rest of the ultrasound findings is described in table 2. 
Table 1. Clinical and demographic variables

\begin{tabular}{|c|c|}
\hline Variable & $n=75$ \\
\hline Age years, mean (SD) & $44.6(14.5)$ \\
\hline Female, $\mathrm{n}(\%)$ & $33(44)$ \\
\hline Weight kg, mean (SD) & $82.06(20.46)$ \\
\hline Height $\mathrm{cm}$, mean (SD) & $164.2(10.2)$ \\
\hline BMI, mean (SD) & $30.4(6.4)$ \\
\hline $\begin{array}{l}\text { Time since psoriasis diagnosis years, } \\
\text { median (IOR) }\end{array}$ & $6(8)$ \\
\hline Plaque psoriasis, n (\%) & $64(85.3)$ \\
\hline Nail involvement, $\mathrm{n}(\%)$ & $36(48)$ \\
\hline Limb involvement, $\mathrm{n}(\%)$ & $38(50.6)$ \\
\hline Scalp involvement, $\mathrm{n}(\%)$ & $22(29.3)$ \\
\hline Diabetes mellitus, $\mathrm{n}(\%)$ & $10(13.3)$ \\
\hline Hypertension, n (\%) & $13(17.3)$ \\
\hline Dyslipidemia, n (\%) & $16(21.3)$ \\
\hline Cutaneous treatment, $\mathrm{n}(\%)$ & 70 (93.3) \\
\hline Systemic treatment, $\mathrm{n}(\%)$ & $22(29.3)$ \\
\hline Methotrexate use, n (\%) & $16(21.3)$ \\
\hline Biologic therapy use, $n(\%)$ & $6(8)$ \\
\hline PASI, median (IOR) & $5.4(8.4)$ \\
\hline PDI, mean (SD) & $0.94(0.08)$ \\
\hline NAPSI, median (IQR) & $4(15)$ \\
\hline ToPAS, median (IQR) & $5(3)$ \\
\hline ToPAS score $>8, n(\%)$ & $12(16)$ \\
\hline IBP, n (\%) & $7(9.3)$ \\
\hline MASES, median (IOR) & $0(0)$ \\
\hline
\end{tabular}

SD: standard deviation, BMI: body mass index, IQR: interquartile range, PASI: Psoriasis Area Severity Index, PDI: Psoriasis Disability Index, NAPSI: Nail Psoriasis Severity Index, ToPAS: Toronto Psoriatic Arthritis Screening, IBP: inflammatory back pain, MASES: Maastricht Ankylosing Spondylitis Enthesitis Score

The image evaluation (ultrasound [US] and MRI) as well as the detailed description of patients with symptoms are described in the supplementary material.

\section{Discussion}

Despite the loss of 33 patients in the study, the systematic protocol detected 26 of 75 patients with musculoskeletal involvement (See Step 3 in Figure 1). The evaluation contributed to the early detection of inflammation in psoriasis patients with and without symptoms.

Evaluation in patients without musculoskeletal symptoms: this is, to the best of our knowledge, the first report of a systematic evaluation protocol of psoriasis patients with and without musculoskeletal symptoms. In a systematic review, to determine the prevalence of underdiagnosed PsA, 394 studies with high heterogeneity $(I(2)=96.86 \%)$ were evaluated, and a prevalence of $15.5 \%$ was found ${ }^{13}$.

There are several reports of musculoskeletal involvement detection using different imaging methods. Recently, Faustini et al. evaluated 55 asymptomatic patients with psoriasis and found that $47 \%$ had at least one hand inflammatory lesion by MRI ${ }^{14}$. We find a similar prevalence $(55 \%)$ of asymptomatic disease in patients with psoriasis. Unlike the previous study, in the evaluation of this study, we consider enthesis as the first musculoskeletal site affected after the skin in patients with psoriasis ${ }^{15}$. In 2008, Raza et al. reported the detection and treatment response of joint disease in asymptomatic patients with psoriasis by scintigraphy scanning. They found a prevalence of joint disease of $70 \%{ }^{16}$. Furthermore, the use of other methods has been evaluated by Takata, et al. They found 18 (33\%) patients with psoriasis vulgaris, the evidence of subclinical PsA, by positron emission tomography/computed tomography using 18 F-fluorodeoxyglucose ${ }^{17}$.

There are reports that underestimate the prevalence of enthesopathy in patients with psoriasis. Oguz, et al. found ultrasound abnormalities in $30 \%$ of patients with psoriasis, but no significant difference compared to controls $^{18}$.

Naredo, et al. observed that synovitis and enthesopathy by US were significantly more frequent in psoriatic patients than in healthy controls. Entheseal PDUS was found in $10(7.4 \%)$ of psoriatic patients, whereas no controls showed this finding. PDUS has been shown to be specific for peripheral enthesitis in spondyloarthritis ${ }^{19}$.

Evaluation of patients with musculoskeletal symptoms: in our study, even though the goal was not to evaluate the diagnostic performance of ToPAS, it was useful to discriminate symptomatic from asymptomatic patients. In previous studies, ToPAS had a $76.6 \%$, $29.7 \%$, and 0.554 sensitivity, specificity, and area under the ROC, respectively ${ }^{3}$. Positive ToPAS was present in $12 / 75(16 \%)$ psoriasis patients. Subsequently, we confirmed joint inflammation in 11/12 of those positive ToPAS patients by ultrasound. This possibly allows us to consider the ToPAS as an initial screening tool in 
Table 2. Entheseal abnormalities by ultrasound

\begin{tabular}{|c|c|c|c|c|c|c|}
\hline MASEI score results & First evaluator & Second evaluator & p & k & \multicolumn{2}{|c|}{ Correlation coefficient } \\
\hline $\begin{array}{l}\text { Enthesopathy } \\
\text { MASEI > 20, n (\%) }\end{array}$ & $6(22.2)$ & $15(55.6)$ & 0.013 & 0.37 & & $\stackrel{\infty}{\circ}$ \\
\hline Power Doppler signal in any enthesis, $n(\%)$ & $13(48.1)$ & $16(59.3)$ & & 0.77 & & $\gtrsim$ \\
\hline Total MASEI, median (IOR) & $16(8)$ & $21(11)$ & & & 0.62 & हᄐ \\
\hline Inflammatory MASEI, median (IOR) & $13(7)$ & $19(5.4)$ & & & 0.57 & (1) \\
\hline Damage MASEI, median (IQR) & $2(5)$ & $3(6)$ & & & 0.63 & \\
\hline
\end{tabular}

MASEI: Madrid Sonographic Enthesitis Index, IQR: interquartile range *Spearman's Rho

patients with psoriasis. We were not able to validate ToPAS 2 in our study, which has performed well in the axial domain ${ }^{20}$. To the best of our knowledge, this is the first study where musculoskeletal effects have been confirmed by imaging in patients with positive ToPAS. To assess diagnostic and therapeutic implications, it is necessary to consider supplementing the entheseal imaging evaluation of these patients.

The prevalence of IBP in our study population was only $7 / 75(9.3 \%)$, and only one had an MRI with sacroiliitis. However, IBP by ASAS criteria could be underdiagnosed in psoriasis patients over 40 years old because of other non-inflammatory LBP causes. It is known that the discriminative value of IBP according to the ASAS criteria is low, with sensitivity, specificity, and balanced accuracy values of 64,50 , and $57 \%$, respectively. Arnbak et al. reported that the prevalence of IBP relative to axial spondyloarthritis according to the ASAS criteria ranged from $10 \%$ for "pain worst in the morning" to $79 \%$ for "morning stiffness ${ }^{21}$." We balanced this disadvantage using MRI in all patients with IBP, and we corroborated axial involvement by MRI only in (1/7) $14.2 \%$ of patients with IBP. In the other six patients, we found other non-inflammatory causes of lumbar pain (degenerative disc disease and facet joint arthrosis). Thom et al. reported that people with psoriasis and PsA had a significantly higher prevalence of axial pain, as measured using the Berlin criteria, compared with people without the disease $(31.1 \%$ vs. $18.9 \%)^{22}$. This higher prevalence could not be compared in our study due to differences in the questionnaires used, the studied population, and other musculoskeletal comorbidities.

Early PsA: a continuum of skin and joint manifestations. The presence of enthesitis could be underdiagnosed unless ultrasound is used as demonstrated in our study. As shown in figure 1, in Group 3, we found that more than half of the patients with asymptomatic psoriasis had evidence of some abnormality in the musculoskeletal ultrasound of their enthesis. Although we accept that our study has the weakness of having lost $32 / 59(54.2 \%)$ of its patients in this group only for performing the ultrasound, all patients were evaluated clinically. According to Gisondi et al., they observed that the thickness of all tendons examined in asymptomatic psoriasis patients was significantly higher than controls ${ }^{23}$. It has been reported by Aydin et al. that the ultrasound appearances of subclinical enthesitis in psoriasis differ from subclinical enthesitis in PsA, with PsA patients having more PD. They found a significant difference between PD positivity in at least one entheseal site in PsA (36.2\%) versus psoriasis $(9.5 \%)^{24}$. We did not compare the thickness of the tendons between asymptomatic patients and ToPAS positive patients because we performed different ultrasound protocols.

In our study, it seemed that the presence of enthesopathy and psoriasis was able to occur simultaneously in a high number of psoriasis patients. Since the frequency of enthesitis detected by ultrasound is greater than symptomatic synovitis, we might think that cutaneous psoriasis and enthesitis have some similar pathophysiological mechanisms.

The patient's follow-up: the patients with a positive ToPAS score ( $\geq 8$ ) underwent a joint ultrasound. The ultrasound study was only performed on 11 of the 12 patients because one of the patients did not attend their appointment. In the group with ToPAS a score of $<8,32$ patients missed their appointments for the echography study. It is possible that those patients were not interested in the study because they were fully asymptomatic by ToPAS and also did not have 

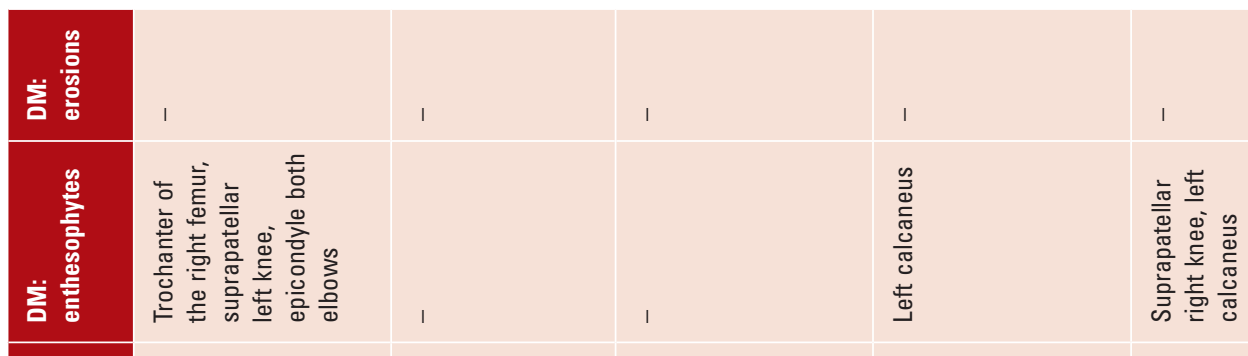

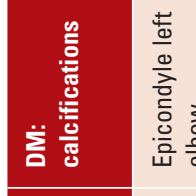

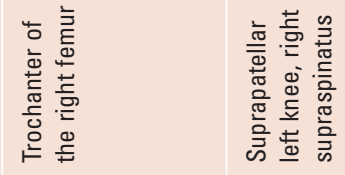

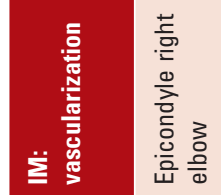

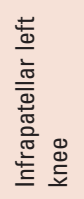

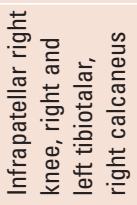

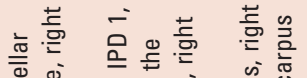

选 这

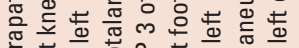

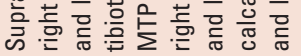

总

樆

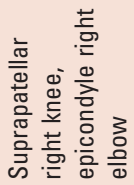

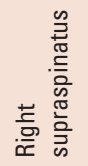

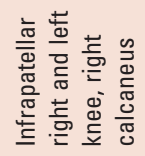

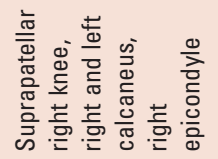

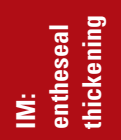
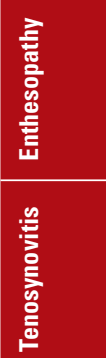

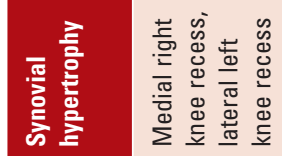

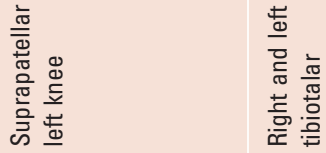

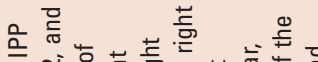

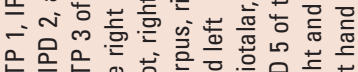

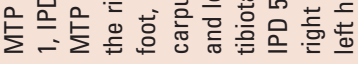

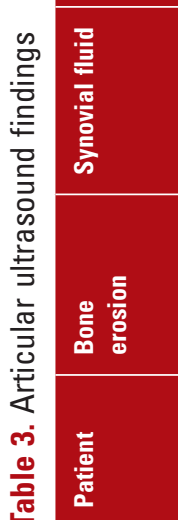

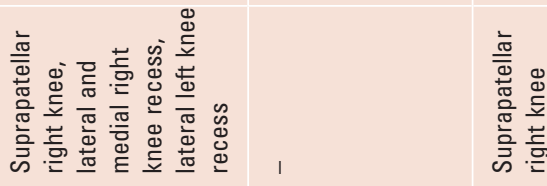

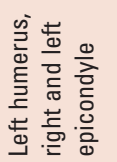

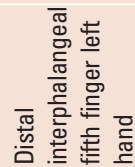

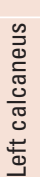

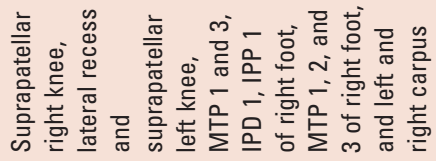

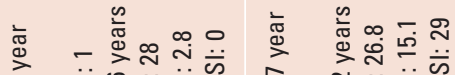

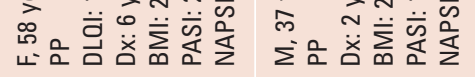

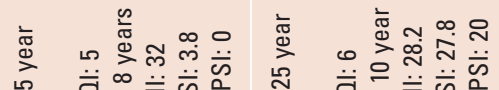

$\varlimsup_{\infty}^{\infty} \infty$

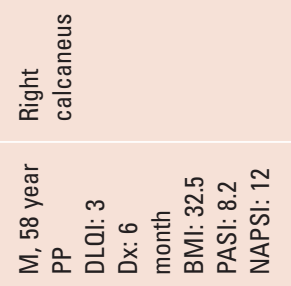




\section{言产譥}

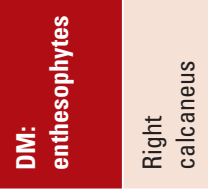
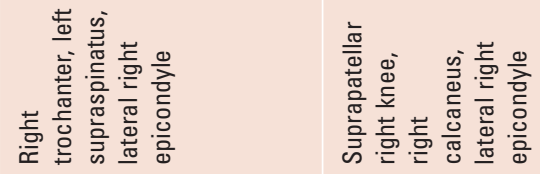

言

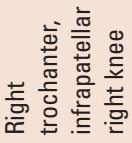

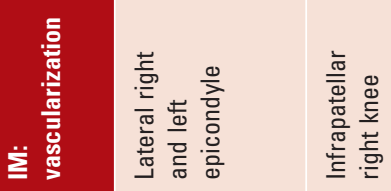

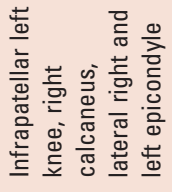

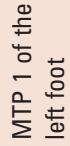

咅兽

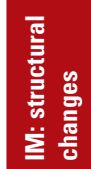

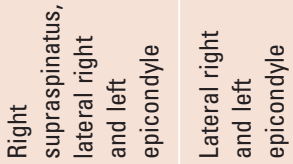

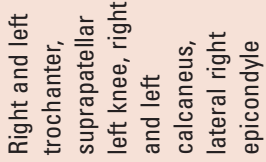

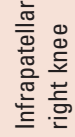
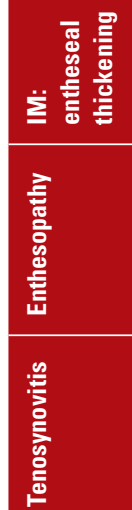

$\overline{\mathrm{a}}$

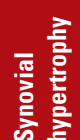

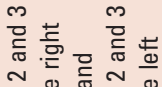

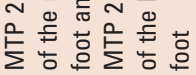

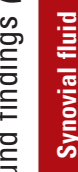

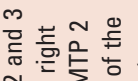

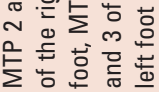

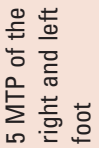
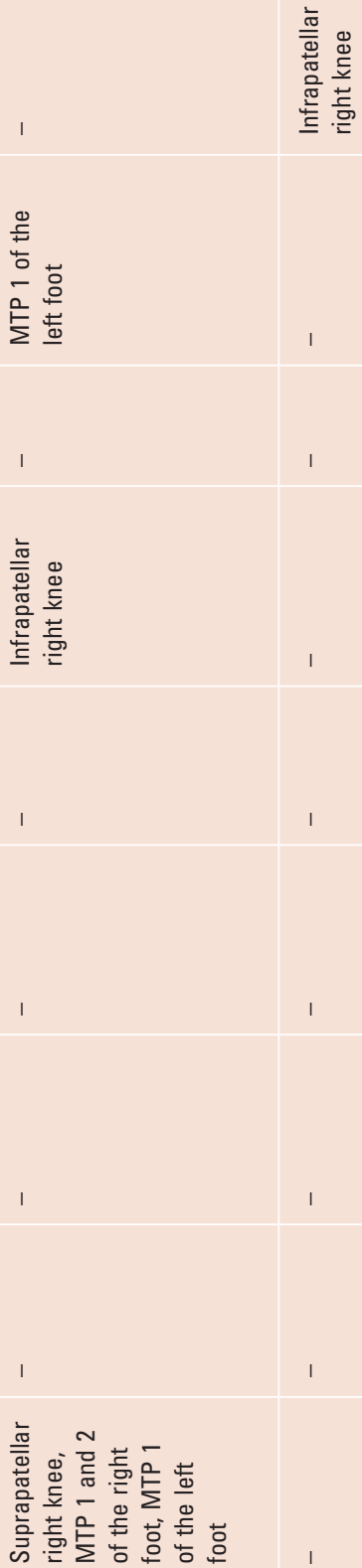

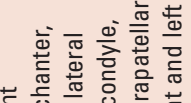

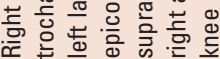

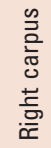

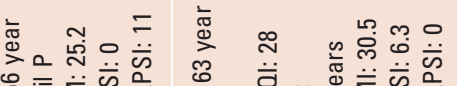

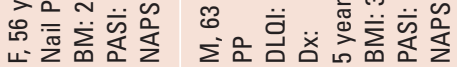

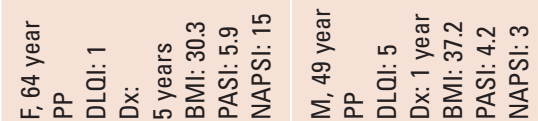


Table 4. MRI findings.

\begin{tabular}{|l|l|}
\hline Patients & MRI \\
\hline $\begin{array}{l}\text { F, 55 years. DLOI 14, Dx: 25 years } \\
\text { Plaque psoriasis, BMI: 26.9, PASI 4.4, NAPSI } 8\end{array}$ & $\begin{array}{l}\text { Hyperintense area on the right iliac toward the lower end of the joint, subchondral } \\
\text { type. In lumbar assessment: disc protrusions with dehydration at L4-L5, L5-S1 }\end{array}$ \\
$\begin{array}{l}\text { M, 35 years. DOLI 1, Dx: } 2 \text { years } \\
\text { Plaque psoriasis, BMI 23.6, PASI 4.6, NAPSI } 41\end{array}$ & $\begin{array}{l}\text { No inflammatory changes in the sacroiliac joints. In lumbar assessment: changes } \\
\text { in intensity of intervertebral disk L5-S1 in relation to changes dehydration and } \\
\text { bulging annulus with partial breakage }\end{array}$ \\
\hline $\begin{array}{l}\text { M, 37 years, DLOI 11, Dx: 2 years } \\
\text { Plaque psoriasis, BMI: 26.8, PASI 15.1, NAPSI } 29\end{array}$ & $\begin{array}{l}\text { No inflammatory changes in the sacroiliac joints. Lumbar evaluation: decreased } \\
\text { signal intensity of the L5-S1 intervertebral disc in relation to changes dehydration } \\
\text { and subsequent small protrusion and central component partially obliterating the } \\
\text { previous epidural space }\end{array}$ \\
\hline $\begin{array}{l}\text { M, 37 years, DLOI 5, Dx: 5 years } \\
\text { Plaque psoriasis, BMI: 24.4, PASI 3.5, NAPSI 17 }\end{array}$ & $\begin{array}{l}\text { No inflammatory changes in the sacroiliac joints. Intervertebral disc L5-S1 with } \\
\text { loss of height and signal intensity changes regarding dehydration }\end{array}$ \\
\hline $\begin{array}{l}\text { F, 27 years, DLOI 3, Dx: 10 years } \\
\text { Nail psoriasis, BMI: 23.2, PASI 0.6, NAPSI 27 }\end{array}$ & $\begin{array}{l}\text { No inflammatory changes in the sacroiliac joints. Disc degeneration and protrusion } \\
\text { of the fibrous ring at L4-L5 and L5 S1 }\end{array}$ \\
\hline $\begin{array}{l}\text { M, } 58 \text { years, DLOI 6, Dx: } 9 \text { years } \\
\text { Plaque psoriasis, BMI: 28.6, PASI 4.8, NAPSI 28 }\end{array}$ & $\begin{array}{l}\text { The sacroiliac joints bilaterally present sclerosis and increased space in the right } \\
\text { sacroiliac joint. The left sacroiliac joint with increased intensity in their articular } \\
\text { margins to consider acute inflammatory changes. In the lumbar evaluation: } \\
\text { decrease in signal intensity of the L5-S1 intervertebral disc in relation to changes } \\
\text { by dehydration plus image with rupture disc protrusion annulus fibrosus }\end{array}$ \\
\hline $\begin{array}{l}\text { F, 27 years, DLOI 2, Dx: 1 year } \\
\text { Nail psoriasis, BMI: 18.8, PASI 9, NAPSI 12 }\end{array}$ & $\begin{array}{l}\text { No inflammatory changes in the sacroiliac joints } \\
\text { Rectification of the lumbar lordosis in the first lumbar segments to consider } \\
\text { changes component of muscle spasm }\end{array}$ \\
\hline
\end{tabular}

F: female, M: male, DLOI: Dermatology Life Quality Index, Dx: time since diagnosis, BMI: body mass index, PASI: Psoriasis Area and Severity Index, NAPSI: Nail Psoriasis Severity Index, MRI: magnetic resonance imaging

IBP. If this was the reason, it is unlikely to affect our results about the prevalence of enthesitis. The low patient response to evaluations has been addressed in other studies before, with patient losses as high as $80 \% 25$.

Ultrasound reliability: Naredo et al..$^{19}$ demonstrated a good interobserver reliability for assessing synovitis and enthesopathy in previous studies. Kendall's W coefficient was 0.64 for the assessment of synovial fluid and synovial hypertrophy signals and 0.85 for the PD signal. In our study, we observed that the interobserver kappa index for detecting enthesitis by MASEI index was 0.33 , while the kappa index for the PD signal was 0.77 . This interobserver reliability is slightly lower than reported in other centers with greater ultrasound experience. However, there is a good correlation with the PD signal in our study.

\section{Limitations}

Nonetheless, we did not perform this study with control patients to establish if the entheseal abnormalities were different to what we found in psoriasis patients, nor did we compare entheseal ultrasounds among the three groups.
In our opinion, the evaluation of patients with psoriasis for MSK involvement should be predominantly clinical, but periodic ultrasound evaluation could be recommended if the ToPAS clinical tool cannot be used first. The use of other imaging methods will be more expensive (PET scan, scintigraphy, and MRI).

\section{Future agenda}

The implication of the inflammatory changes evidenced by ultrasounds in asymptomatic patients could be addressed in future studies. As noted before, an early diagnosis accounts for a better prognosis in all inflammatory arthropathies. However, what would be the best way to treat these asymptomatic patients? How would the follow-up be recommended? With the use of ultrasounds? Even though the enthesitis (by Power Doppler) is a prognostic lesion, what is the prognostic value of the other entheseal lesions demonstrated? In the context of a patient with already diagnosed PsA, could the presence of enthesitis be an anti-TNF indication? What would be the treatment of these patients be? Could the systemic treatment of a psoriasis patient prevent the development of MSK involvement? Is this the reason why patients with less 
skin involvement (ergo less treatment) develop more MSK involvement?

\section{Conclusion}

This systematic and steered approach is useful for hidden inflammatory MSK involvement in psoriasis patients. Enthesitis is a very common finding by ultrasound in psoriasis patients. We propose a close follow-up of patients with psoriasis with entheseal abnormalities by ultrasound for early diagnosis of PsA. The prognostic and treatment implications should be addressed in future studies.

\section{Acknowledgments}

We thank Daniel Oswaldo Treviño-Montes and Brenda Roxana Vázquez-Fuentes for their help in patient recruiting and study monitoring. We thank Brenda Tovar-Jasso for patient following appointment coordination. We thank Ericka Pérez-Hernández for ultrasound assitstance.

\section{Funding}

This study was funded by Abbvie Laboratories for the MRI payment.

\section{Conflict of interest}

The authors declare they have no conflicts of interest.

\section{Ethical disclosures}

Protection of human and animal subjects. The authors declare that the procedures followed were in accordance with the regulations of the relevant clinical research ethics committee and with those of the Code of Ethics of the World Medical Association (Declaration of Helsinki).

Confidentiality of data. The authors declare that they have followed the protocols of their work center on the publication of the patient data.

Right to privacy and informed consent. The authors have obtained the written informed consent of the patients or subjects mentioned in the article. The corresponding author is in possession of this document.

\section{References}

1. Villani AP, Rouzaud M, Sevrain M, et al. Symptoms dermatologists should look for in daily practice to improve detection of psoriatic arthritis in psoriasis patients: an expert group consensus. J Eur Acad Dermatol Venereol. 2014;28:S27-32.

2. Tintle SJ, Gottlieb AB. Psoriatic arthritis for the dermatologist. Dermatol Clin. 2015;33:127-48.

3. Coates LC, Aslam T, Al Balushi F, et al. Comparison of three screening tools to detect psoriatic arthritis in patients with psoriasis (CONTEST study). Br J Dermatol. 2013;168:802-7.

4. Langley RG, Ellis CN. Evaluating psoriasis with psoriasis area and severity index, psoriasis global assessment, and lattice system physician's global assessment. J Am Acad Dermatol. 2004;51:563-9.

5. Finlay AY, Kelly SE. Psoriasis--an index of disability. Clin Exp Dermatol. 1987:12:8-11.

6. Finlay AY, Khan GK. Dermatology life quality index (DLQI)--a simple practical measure for routine clinical use. Clin Exp Dermatol. 1994; 19:210-6.

7. Rich P, Scher RK. Nail psoriasis severity index: a useful tool for evaluation of nail psoriasis. J Am Acad Dermatol. 2003;49:206-12.

8. Chandran V, Gladman DD. Toronto psoriatic arthritis screening (ToPAS) questionnaire: a report from the GRAPPA 2009 annual meeting. J Rheumatol. 2011;38:546-7.

9. Sieper J, van der Heijde D, Landewe R, et al. New criteria for inflammatory back pain in patients with chronic back pain: a real patient exercise by experts from the Assessment of Spondylo Arthritis international Society (ASAS). Ann Rheum Dis. 2009;68:784-8.

10. Heuft-Dorenbosch L, Spoorenberg A, van Tubergen A, et al.Assessment of enthesitis in ankylosing spondylitis. Ann Rheum Dis. 2003;62:127-32.

11. Wakefield RJ, Balint PV, Szkudlarek M, et al. Musculoskeletal ultrasound including definitions for ultrasonographic pathology. J Rheumatol. 2005;32:2485-7.

12. de Miguel $E$, Cobo $T$, Muñoz-Fernández $S$, et al. Validity of enthesis ultrasound assessment in spondyloarthropathy. Ann Rheum Dis. 2009; 68:169-74.

13. Villani AP, Rouzaud M, Sevrain M, et al. Prevalence of undiagnosed psoriatic arthritis among psoriasis patients: systematic review and meta-analysis. J Am Acad Dermatol. 2015;73:242-8.

14. Faustini F, Simon D, Oliveira I, et al. Subclinical joint inflammation in patients with psoriasis without concomitant psoriatic arthritis: a cross-sectional and longitudinal analysis. Ann Rheum Dis. DOI: 10.1136/annrheumdis-2015-208821.

15. Girolomoni G, Gisondi P. Psoriasis and systemic inflammation: underdiagnosed enthesopathy. J Eur Acad Dermatol Venereol. 2009;23S1:3-8.

16. Raza N, Hameed A, Ali MK. Detection of subclinical joint involvement in psoriasis with bone scintigraphy and its response to oral methotrexate. Clin Exp Dermatol. 2008:33:70-3.

17. Takata T, Takahashi A, Taniguchi $Y$, et al. Detection of asymptomatic enthesitis in psoriasis patients: an onset of psoriatic arthritis? J Dermatol. DOI: $10.1111 / 1346-8138.13212$.

18. Oguz ID, Gul U, Koparal SS, et al. Investigation of enthesopathy with ultrasonography and comparison with skin findings in asymptomatic psoriatic patients. Dermatology. 2016;232:312-8.

19. Naredo E, Moller I, de Miguel E, et al. High prevalence of ultrasonographic synovitis and enthesopathy in patients with psoriasis without psoriatic arthritis: a prospective case-control study. Rheumatology (Oxford). 2011:50:1838-48.

20. Tom BD, Chandran V, Farewell VT, et al. Validation of the toronto psoriatic arthritis screen version 2 (ToPAS 2). J Rheumatol. 2015;42:841-6.

21. Arnbak $B$, Hendricks $O$, Horslev-Petersen $K$, et al. The discriminative value of inflammatory back pain in patients with persistent low back pain. Scand J Rheumatol. 2016;45:321-8.

22. Thom N, Ritchlin CT, Zhang X, et al. Prevalence of chronic axial pain, inflammatory back pain, and spondyloarthritis in diagnosed psoriasis. Arthritis Care Res (Hoboken). 2015;67:829-35.

23. Gisondi P, Tinazzi I, El-Dalati G, et al. Lower limb enthesopathy in patients with psoriasis without clinical signs of arthropathy: a hospital-based case-control study. Ann Rheum Dis. 2008;67:26-30.

24. Aydin SZ, Ash ZR, Tinazzi I, et al. The link between enthesitis and arthritis in psoriatic arthritis: a switch to a vascular phenotype at insertions may play a role in arthritis development. Ann Rheum Dis. 2013; 72:992-5.

25. Coates LC, Savage L, Waxman R, et al. Comparison of screening questionnaires to identify psoriatic arthritis in a primary care population: a cross-sectional study. Br J dermatol. DOI: 10.1111/bjd.14604. 